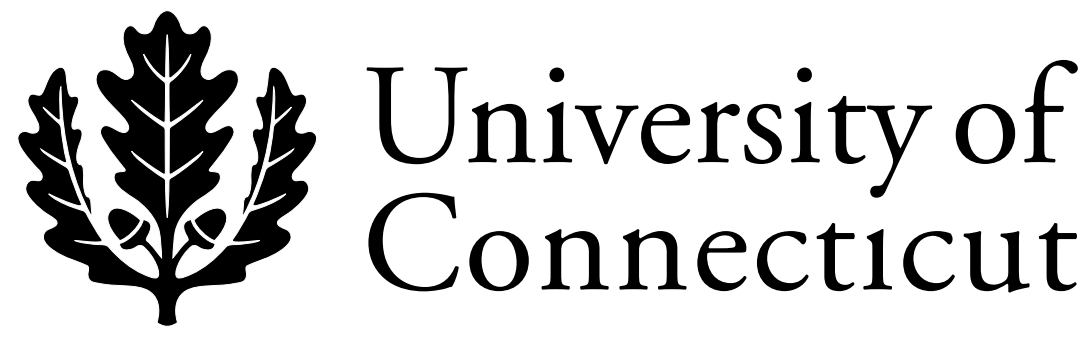

Department of Economics Working Paper Series

\title{
Continuous Lexicographic Preferences
}

Vicki Knoblauch

University of Connecticut

Working Paper 2003-31

August 2003

341 Mansfield Road, Unit 1063

Storrs, CT 06269-1063

Phone: (860) 486-3022

Fax: (860) 486-4463

http://www.econ.uconn.edu/ 


\begin{abstract}
Under what conditions are lexicographically representable preferences continuously representable? This question is actually two questions, since there are two natural definitions of continuity for lexicographic representations. A complete answer is given for one of these questions, and the other is answered for two-dimensional lexicographic representations.
\end{abstract}

Journal of Economic Literature Classification: D11, D70

Keywords: preferences, lexicographic order, continuous representations 


\section{Introduction}

Most of the work in Debreu's (1964) characterization of preferences representable by a continuous utility function involves showing that every binary relation that can be represented by a utility function can be represented by a continuous utility function. Theorems 1 and 2 below address the related question of continuous representability for lexicographically representable preferences.

The lexicographic order on $\Re^{n}$ favors one point of $\Re^{n}$ over another if the points are distinct and, in the first coordinate at which they differ, the first point exceeds the second. It is sometimes argued that a reasonable individual's preferences cannot be lexicographic, since reasonable preferences should be representable by a utility function. This argument cannot be made for group preferences. Suppose a group employs a reasonable decisionmaking procedure-voting-each time it is presented with a pair of alternatives from $\Re^{n}$. Then, even if each member of the group holds preferences generated by a utility function, the group's preferences might be lexicographic, Paretian or even cyclic.

A lexicographic representation for preferences over a set $X$ is a function from $X$ to $\Re^{n}$ ordered by the lexicographic order that preserves preference and indifference. Consider a team of $n$ executives, each of whom holds preferences governed by a utility function over a set $X$. Each day the top executive is presented with a pair of alternatives from $X$ and is asked to choose one. She does so unless she is indifferent between the two alternatives, in which case she passes the choice on to her second in command. The procedure is repeated until the choice is made or the pair has been passed by all $n$ executives. Depending on the individual preferences of executives, the team preferences as defined by the decision procedure may or may not be representable by a utility function, but the team preferences do have a lexicographic representation. The required function from $X$ to $\Re^{n}$ has as its $i$ th coordinate function the $i$ th executive's utility function.

A preference representation provides a conceptual handle for an individual's or group's preferences by embedding those preferences in another better-known preference profile, such as $>$ on $\Re$, the lexicographic order on $\Re^{n}$ or the Pareto relation on $\Re^{n}$. Adding continuity further enhances understanding. A continuous lexicographic order provides not 
only a portrait of preference but also a portrait of closeness of alternatives. In addition, continuity can be a useful technical tool; it can for example be helpful in determining which subsets of a set of alternatives contains a maximally preferred element.

A lexicographic representation is said to be continuous if it is continuous as a function from $X$ with the given order topology to $\Re^{n}$ with the lexicographic order topology; it is said to be coordinate continuous if its coordinate functions are continuous functions from $X$ with the given order topology to $\Re$ with the Euclidean topology. Propositions 1 and 2 below concern these definitions, and serve as the basis for a short discussion comparing the two definitions.

The main results of this paper comprise easy tests of the continuous representability of lexicographic preferences: lexicographically representable preferences are continuously representable if and only if they are locally utility representable (Theorem 1); and preferences lexicographically represented in $\Re^{2}$ are coordinate-continously representable if and only if the points at which they are not locally utility representable satisfy two countability conditions to be introduced below (Theorem 2).

Theorems 1 and 2 can be thought of as modules that can be combined with any lexicographic representation existence theorem (such as Knoblauch [2000]) to obtain a continuous lexicographic representation existence theorem and a coordinate continuous lexicographic representation existence theorem.

Lexicographic preferences appear in both theoretical and empirical studies. Fishburn (1975) characterizes lexicographic orders axiomatically. Binmore and Samuelson (1992) study games wherein a player's utility is lexicographic in his payoff and the simplicity of the strategy he chooses. As an example of an empirical study, Jensen (1990) tests fertility decisions to determine whether preferences are lexicographic with old-age security as the primary deciding factor. Most recently, Hougaard and Tvede (2001) use generalized lexicographic relations to construct an example of an economy with continuous demand functions that nevertheless does not possess equilibria.

The paper is organized as follows. Section 2 consists of preliminaries, a discussion of two definitions of continuity for lexicographic representations, and the statements of two theorems on the existence of continuous lexicographic representations. Section 3 contains 
four examples illustrating the theorems, which are proven in Section 4.

\section{Statement of Results}

A binary relation on a set $X$ is a subset of $X \times X$. If $\succ$ is a binary relation on $X$, $<x, y>\in \succ$ will be written $x \succ y$. If $\succ$ is a binary relation on $X$, then $\succsim$ and $\sim$ are the binary relations defined by $x \succsim y$ if $\operatorname{not}(y \succ x)$ and $x \sim y$ if $x \succsim y \succsim x$. For $x \in X$ let $W(x)=\{y \in X: x \succ y\}$ and let $B(x)=\{y \in X: y \succ x\}$.

The $\succ$-order topology on $X$ is the topology generated by the subbasis consisting of all sets $W(x)$ and all sets $B(x)$ where $x$ is allowed to vary over $X$.

The lexicographic order $>_{L}$ on $\Re^{n}$ is defined by $x>_{L} y$ if there exists $k \in\{1,2, \ldots, n\}$ such that $x_{i}=y_{i}$ for $i<k$, and $x_{k}>y_{k}$.

A lexicographic representation for a binary relation $\succ$ on a set $X$ is a function $v: X \rightarrow$ $\Re^{n}$ such that, for $x, y \in X, x \succ y$ if and only if $v(x)>_{L} v(y)$.

A lexicographic representation is continuous if it is continuous as a function from $X$ with the $\succ$-order topology to $\Re^{n}$ with the lexicographic order topology.

A binary relation $\succ$ on $X$ is locally utility representable at $x \in X$ if there is an open $O \subseteq X$ with $x \in O$ such that $\left.\succ\right|_{O}$ has a utility representation. Here $\left.\succ\right|_{O}$ is $\succ \cap(O \times O)$, the restriction of $\succ$ to $O$. Also, $\succ$ is locally utility representable if it is locally utility representable at $x$ for every $x \in X$.

Theorem 1. A lexicographically representable binary relation has a continuous lexicographic representation if and only if it is locally utility representable

A preference representation $v: X \rightarrow \Re^{n}$ is coordinate continuous if for each $i \in$ $\{1,2, \ldots, n\} v_{i}$ is a continuous function from $X$ with the $\succ$-order topology to $\Re$ with the Euclidean topology.

A binary relation $\succ$ on $X$ is one-sided locally utility representable at $x \in X$ if there is an open $O \subseteq X$ with $x \in O$ such that either $\left.\succ\right|_{O \cap W(x)}$ or $\left.\succ\right|_{O \cap B(x)}$ can be represented by a utility function. Also, $\succ$ is exactly one-sided locally utility representable at $x$ if $\succ$ is one-sided locally utility representable at $x$, but not locally utility representable at $x$. 
Theorem 2. A binary relation $\succ$ on $X$ with a lexicographic representation $v: X \rightarrow \Re^{2}$ has a coordinate continuous lexicographic representation if and only if

a. the set of elements of $X$ at which $\succ$ is exactly one-sided locally utility representable has only countably many -equivalence classes.

b. there is a nested sequence $<A^{k}>$ of open subsets of $X$ such that $\bigcap_{k=1}^{+\infty} A^{k}$ is the set of elements of $X$ at which $\succ$ is not locally utility representable.

Four examples will serve to illustrate the theorems, but first two propositions will facilitate a comparison of continuity and coordinate continuity.

Proposition 1. A preference representation $v: X \rightarrow \Re^{n}$ for $\succ$ on $X$ is coordinate continuous if and only if it is continuous as a function from $X$ with the $\succ$-order topology to $\Re^{n}$ with the Euclidean topology.

Proof. Suppose $v$ is coordinate continuous and $O \subseteq \Re^{n}$ is open in the Euclidean topology. Then $O$ is a union of sets of the form $I^{1} \times I^{2} \times \ldots \times I^{n}$ where each $I^{i}$ is an open interval in $\Re$. Then $v^{-1}\left(I^{1} \times I^{2} \times \ldots \times I^{n}\right)=\bigcap_{i=1}^{n} v_{i}^{-1}\left(I^{i}\right)$ which is open. Therefore $v^{-1}(O)$ is open which implies $v$ is continuous as a function from $X$ to $\Re^{n}$ with the Euclidean topology.

Now suppose $v$ is continuous as a function from $X$ to $\Re^{n}$ with the Euclidean topology, $O$ is an open subset of $\Re$ and $i \in\{1,2, \ldots, n\}$. Then $v_{i}^{-1}(O)=v^{-1}(\Re \times \ldots \times \Re \times O \times \Re \times \ldots \times$ $\Re)$ where $O$ occurs in the $i$ th factor. Since $v$ is continuous, $v^{-1}(\Re \times \ldots \times \Re \times O \times \Re \times \ldots \times \Re)$ is open so that $v_{i}^{-1}(O)$ is open and $v$ is coordinate continuous.

Proposition 2. A continuous lexicographic representation is coordinate continuous.

Proof. Suppose $v: X \rightarrow \Re^{n}$ is a continuous lexicographic representation for $\succ$ on $X$ and $O \subseteq \Re^{n}$ is open in the Euclidean topology. Then $O$ is open in the lexicographic order topology, since $r \in O$ implies the existence of $a, b \in \Re$ such that $r \in O^{\prime}=$ $\left\{<r_{1}, r_{2}, \ldots, r_{n-1}, s>: a<s<b\right\} \subseteq O$. Since $v$ is continuous, $v^{-1}(O)$ is open. This proves that $v$ is continuous as a function from $X$ to $\Re^{n}$ with the Euclidean topology. By 
Proposition 1, $v$ is coordinate continuous.

Proposition 1 can be used to argue that, for lexicographic representations, coordinate continuity is a less appropriate concept than continuity; a lexicographic representation links a binary relation $\succ$ with the lexicographic order on $\Re^{n}$, so it is reasonable that continuity for a lexicographic representation should link the $\succ$-order topology on $\Re^{n}$ with the lexicographic order topology on $\Re^{n}$, not with the Euclidean order topology on $\Re^{n}$.

On the other hand, coordinate continuity suggests the executive team scenario of Section 1, with the added feature that each executive has preferences represented by a continuous utility function. Under this scenario coordinate continuity seems to be an appropriate definition of continuity for lexicographic representations.

Notice that coordinate continuity requires that each executive's utility function is continuous as a function from $X$ with the given $\succ$-order topology, not merely as a function from $X$ with the order topology generated by the executive's own preferences.

This third form of continuity could be called weak coordinate continuity. There are two reasons for not further considering weak coordinate continuity. First, the relationship between a given binary relation $\succ$ and the topology on $X$ generated by a coordinate of a lexicographic representation for $\succ$ could best be described as tenuous. Second, by Debreu (1964) every lexicographically representable binary relation has a weakly coordinatecontinuous lexicographic representation. Therefore there is no need for a theorem characterizing lexicographically representable binary relations that are weakly coordinatecontinuously lexicographically representable, and consequently no need for examples illustrating such a theorem.

\section{Four Examples.}

The following examples illustrate Theorems 1 and 2 .

The binary relation of Example 1 has neither a continuous nor a coordinate-continuous lexicographic representation. 
Example 1. Let $X=(0,1] \times\{0,1\} \subseteq \Re^{2}$ and define $\succ$ on $X$ by $x \succ y$ if $x>_{L} y$. Obviously $v(x)=x$ is lexicographic representation for $\succ$.

First if $r \in(0,1]$ then $O=W(<r, 1>)$ is open, $<r, 0>\in O$ and $O \cap B(<r, 0>)=\emptyset$ so that, trivially, $>\left.\right|_{O \cap B(<r, 0>)}$ can be represented by a utility function.

Next suppose $r \in(0,1], O \subseteq X$ is open, $<r, 0>\in O$ and $Q \subseteq O$ is $\succ$-dense in $O$, that is, for $x, y \in O, x \succ y$ implies $x \succsim q \succ y$ for some $q \in Q$. Choose $s \in(0, r)$ such that $B(<s, 0>) \cap W(<r, 0>) \subseteq O$. For each $t \in(s, r),<t, 1>\succ<t, 0>,<t, 1>\in O$ and $\langle t, 0\rangle \in O$ so that $\langle t, 1\rangle \succsim q \succ<t, 0>$ for some $q \in Q$. Then $<t, 1\rangle=q$. Therefore $(s, r) \times\{1\} \subseteq Q$ which means $Q$ is uncountable. Since every $\succ$-dense subset of $Q$ is uncountable, by Debreu (1964), $\left.\succ\right|_{O}$ cannot be represented by a utility function.

The previous two paragraphs demonstrate that $\succ$ is exactly one-sided locally utility representable at each point of $\{\langle r, 0\rangle: r \in(0,1]\}$. But clearly for this example if $x \neq y$ then it is not the case that $x \sim y$. Therefore $\{\langle r, 0\rangle: r \in(0,1]\}$ has uncountably many $\sim$-equivalence classes. By Theorem 2, condition (a), $\succ$ does not have a coordinatecontinuous lexicographic representation. By Theorem 1 or Proposition $2, \succ$ does not have a continuous lexicographic representation.

The binary relation of Example 2 has neither a continuous nor a coordinate-continuous lexicographic representation, but this time it is condition (b) of Theorem 2 that is violated.

Example 2. Let $X=Q \times\{0\} \cup J \times(-1,1) \subseteq \Re^{2}$ where $Q$ is the set of rationals in $(0,1)$ and $J$ is the set of irrationals in $(0,1)$. Define $\succ$ on $X$ by $x \succ y$ if $x>_{L} y$. Obviously $v(x)=x$ is a lexicographic representation for $\succ$.

If $\langle j, t>\in J \times(-1,1)$, then $\succ$ is locally utility representable at $\langle j, t>$ since $\left.v\right|_{\{j\} \times(-1,1)}$ is a utility function representing $\left.\succ\right|_{\{j\} \times(-1,1)}$.

If $\langle q, 0>\in Q \times\{0\}$, then by an argument like that in Example 1, $\succ$ is not locally utility representable at $\langle q, 0\rangle$.

Suppose $<A^{k}>$ is a nested sequence of open subsets of $X$ such that $Q \times\{0\} \subseteq A^{k}$ for all $k$. Then $<O^{k}>=<$ Interior $\left(A^{k} \cap(0,1) \times\{0\}\right)>$ is a nested sequence of open subsets of $(0,1) \times\{0\}$ such that $Q \times\{0\} \subseteq O^{k}$ for all $k$. 
By the Baire Category Theorem, $\cap_{k=1}^{+\infty} O^{k} \neq Q \times\{0\}$ [Dugundji (1966),p.249]. Therefore $\cap_{k=1}^{+\infty} A^{k} \neq Q \times\{0\}$. Since condition (b) of Theorem 2 cannot be met, $\succ$ has no coordinate-continuous lexicographic representation. By Proposition 2 or Theorem 1, $\succ$ has no continuous lexicographic representation.

The binary relation of Example 3 has both a continuous and a coordinate continuous lexicographic representation.

Example 3. Let $X=[0,1] \times(0,1) \cup\{<1,1>\} \cup(2,3] \times\{0\} \subseteq \Re^{2}$. Define $\succ$ on $X$ by $x \succ y$ if $x>_{L} y$. Obviously $v(x)=x$ is a lexicographic representation for $\succ$.

Is $\succ$ locally utility representable? If $y \in[0,1] \times(0,1)$, let $O=\left\{y_{1}\right\} \times(0,1)$ and let $u(x)=x_{2}$. If $y \in(2,3] \times\{0\}$ let $O=(2,3] \times\{0\}$ and let $u(x)=x_{1}$. If $y=<1,1>$, let $O=\{1\} \times(0,1] \cup(2,3] \times\{0\}$ and let

$$
u(x)= \begin{cases}x_{2} & \text { if } x_{1}=1 \\ x_{1}, & \text { if } x_{2}=0\end{cases}
$$

Since $\succ$ is locally utility representable, by Theorem $1 \succ$ has a continuous lexicographic representation.

The procedure that will be introduced in the proof of Theorem $1(\Leftarrow)$ produces the following continuous lexicographic representation for $\succ$ :

$$
V(x)=\left\{\begin{array}{cc}
x & \text { if } 0 \leq x_{1} \leq 1 \\
<1, x_{1}-1> & \text { if } 2<x_{1} \leq 3 .
\end{array}\right.
$$

By Proposition 2, $v(x)$ is also a coordinate-continuous lexicographic representation for $\succ$.

The binary relation of Example 4 has a coordinate continuous, but not a continuous, lexicographic representation.

Example 4. Let $X=(0,1) \times(0,1) \cup\{\langle 1,0\rangle\}$. Define $\succ$ on $X$ by $x \succ y$ if $x>_{L} y$. Obviously $v(x)=x$ is a lexicographic representation for $\succ$.

By the argument used in Example 1, $\succ$ is not locally utility representable at $<1,0\rangle$. By Theorem 1, $\succ$ does not have a continuous lexicographic representation. 
If $y \in(0,1) \times(0,1)$, let $O=\left\{y_{1}\right\} \times(0,1)$ and let $u(x)=x_{2}$. This shows that $\succ$ is locally utility representable at every $y \in X-\{<1,0\rangle\}$. Therefore $\succ$ satisfies condition (a) of Theorem 2.

For each positive integer $k$, let $A^{k}=\left\{x \in X: x_{1}>1-\frac{1}{k}\right\}$. Then $<A^{k}>$ is a nested sequence of open subsets of $X$ such that $\left.\cap_{k=1}^{+\infty} A^{k}=\{<1,0\rangle\right\}$ so that $\succ$ satisfies condition (b) of Theorem 2.

By Theorem 2, $\succ$ has a coordinate continuous lexicographic representation. The procedure that will be introduced in the proof of Theorem $2(\Leftarrow)$ produces the following coordinate continuous lexicographic representation for $\succ$ :

$$
V(x)= \begin{cases}<x_{1}, x_{2}\left(1-x_{1}\right)> & \text { for } 0<x_{1}<1 \\ <1,0> & \text { for } x=<1,0>\end{cases}
$$

\section{Proofs of Theorems 1 and 2.}

\section{Proof of Theorem $1(\Rightarrow)$.}

Suppose $\succ$ is a binary relation on $X, v: X \rightarrow \Re^{n}$ is a continuous lexicographic representation for $\succ$ and $x \in X$.

Let $U=\left\{<v_{1}(x), v_{2}(x), \ldots, v_{n-1}(x), r_{n}>: r_{n} \in \Re\right\}$.

Let $O=v^{-1}(U)$. Since $v(x) \in U, x \in O$. Since $U$ is open in the $>_{L^{-}}$order topology and $v$ is continuous, $O$ is open. If $y, z \in O$ then $y \succ z$ if and only if $v(y)>_{L} v(z)$ if and only if $v_{n}(y)>v_{n}(z)$. This establishes $\left.v_{n}\right|_{O}$ as a utility function for $\left.\succ\right|_{O}$. Therefore $\succ$ is locally utility representable.

Proof of Theorem $1(\Leftarrow)$.

The proof proceeds by induction on $n$. When $n=1$, that is, when $\Re^{n}=\Re^{1}$, a lexicographic representation is a utility function. By Debreu (1964) every binary relation represented by a utility function can be represented by a continuous utility function.

Suppose that $n>1$.

Induction Hypothesis: If a binary relation is locally utility representable and has a lexicographic representation from $X$ to $\Re^{n-1}$, then it has a continuous lexicographic represen- 
tation from $X$ to $\Re^{n-1}$.

Suppose also that $\succ$ is a locally utility representable binary relation on $X$ and $v: X \rightarrow$ $\Re^{n}$ is a lexicographic representation for $\succ$.

Fix $r \in \Re$. Let

$$
\begin{aligned}
& D(r)=\left\{s \in \Re: s \geq r \text { and }\left.\succ\right|_{v_{1}^{-1}([r, s])}\right. \text { has a lexicographic representation } \\
&\left.v^{s}: v_{1}^{-1}([r, s]) \rightarrow \Re^{n-1}\right\} .
\end{aligned}
$$

Then $D(r)=[r, d]$ for some $d \geq r$ or $D(r)=[r,+\infty]$ or $D(r)=[r, d)$ for some $d>r$ or $D(r)=\emptyset$.

The alternative $D(r)=\emptyset$ is not possible since, writing $v_{-1}$ for $\left(v_{2}, v_{3}, \ldots, v_{n}\right)$, $\left.v_{-1}\right|_{v_{1}^{-1}([r, r])}$ is an $n-1$ dimensional lexicographic representation for $\left.\succ\right|_{v_{1}^{-1}([r, r])}$.

To see that the alternative $D(r)=[r, d)$ is not possible, suppose $r=d^{1}<d^{2}<\ldots<d$; $d^{k} \rightarrow d$; for $k$ a positive integer, $v^{k}: v_{1}^{-1}\left(\left[r, d^{k}\right]\right) \rightarrow(1-1 / k, 1-1 /(k+1)) \times \Re^{n-2}$ is a lexicographic representation for $\left.\succ\right|_{v_{1}^{-1}\left(\left[r, d^{k}\right]\right)}$; and $v_{-1}: X \rightarrow(1,2) \times \Re^{n-2}$. Then

$$
v^{*}(x)= \begin{cases}v^{k}(x) & \text { if } x \in v_{1}^{-1}\left(\left[d^{k}, d^{k+1}\right)\right) \\ v_{-1}(x) & \text { if } x \in v_{1}^{-1}(\{d\}) .\end{cases}
$$

is an $n-1$ dimensional lexicographic representation for $\left.\succ\right|_{v_{1}^{-1}([r, d])}$.

In summary, $D(r)=[r, d]$ for some $d \geq r$ or $D(r)=[r,+\infty)$. In addition, $\left.\succ\right|_{v_{1}^{-1}(D(r))}$ has an $n-1$ dimensional lexicographic representation: if $D(r)=[r, d]$, an $n-1$ dimensional representation exists by the definition of $D(r)$; if $D(r)=[r,+\infty)$, mimicking the construction of $v^{*}$ produces an $n-1$ dimensional representation.

Next define

$C(r)=\left\{s \in \Re: s \leq r\right.$ and $\left.\succ\right|_{v_{1}^{-1}([s, r])}$ has an $n-1$ dimensional lexicographic representation\}.

If $E(r)=C(r) \cup D(r)$, then $E(r)$ is a closed interval; that is, $E(r)=[c, d],[c,+\infty),(-\infty, d]$ or $(-\infty,+\infty)$, where $c \leq r \leq d$. Also, $\left.\succ\right|_{v_{1}^{-1}(E(r))}$ has an $n-1$ dimensional lexicographic representation. 
If $r, s \in \Re$ and $E(r) \cap E(s) \neq \emptyset$, then it can be shown that $E(r)=E(s)$ using a construction that resembles the construction of $v^{*}$, but splices together only two functions. For example if $a \in E(r) \cap E(s), b \in E(r)$ and $b<a<s$, then an $n-1$ dimensional lexicographic representation for $\left.\succ\right|_{v_{1}^{-1}([b, s])}$ is constructed from $n-1$ dimensional lexicographic representations for $\left.\succ\right|_{v_{1}^{-1}([b, a])}$ and $\left.\succ\right|_{v_{1}^{-1}([a, s])}$. Therefore the $E(r)$ 's form a partition of $\Re$.

Summarizing the proof so far, there is a partition $\left\{E^{\alpha}\right\}$ of $\Re$ such that Each $E^{\alpha}$ is a closed interval

$\left.\succ\right|_{v_{1}^{-1}\left(E^{\alpha}\right)}$ has an $n-1$ dimensional lexicographic representation

If $K \subseteq \Re$ is an interval and $\left.\succ\right|_{v_{1}^{-1}(K)}$ has an $n-1$ dimensional lexicographic representation, then $K \subseteq E^{\alpha}$ for some $E^{\alpha}$

The partition $\left\{E^{\alpha}\right\}$ will now be used to construct a continuous lexicographic representation for $\succ$. Let

$$
V_{1}(x)= \begin{cases}d & \text { if } v_{1}(x) \in E^{\alpha}=[c, d] \text { or }(-\infty, d] \\ c & \text { if } v_{1}(x) \in E^{\alpha}=[c,+\infty] \\ 0 & \text { if } v_{1}(x) \in E^{\alpha}=(-\infty,+\infty) .\end{cases}
$$

For each $E^{\alpha}$, let $v^{\alpha}$ be a continuous $n-1$ dimensional lexicographic representation for $\left.\succ\right|_{v_{1}^{-1}\left(E^{\alpha}\right)}$. An $n-1$ dimensional lexicographic representation exists by (2); and by the induction hypothesis it can be taken to be a continuous function from $v_{1}^{-1}\left(E^{\alpha}\right)$ with the

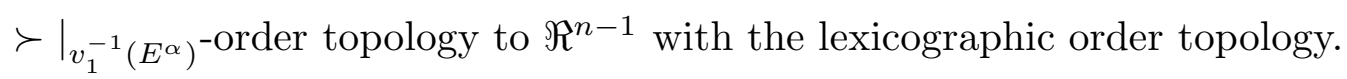

Let $\left(V_{2}(x), V_{3}(x), \ldots, V_{n}(x)\right)=v^{\alpha}(x)$ for that $\alpha$ such that $v_{1}(x) \in E^{\alpha}$. Denote $\left(V_{2}(x), V_{3}(x), \ldots, V_{n}(x)\right)$ by $V_{-1}(x)$.

It remains to show that $V=\left(V_{1}, V_{-1}\right)$ is a continuous lexicographic representation for $\succ$.

Since $v$ is a lexicographic representation for $\succ, x \succ y$ if and only if $v(x)>_{L} v(y)$. By the definition of $V, v(x)>_{L} v(y)$ if and only if $V(x)>_{L} V(y)$. Therefore $V$ is a lexicographic representation for $\succ$.

Next suppose $r \in \Re^{n}$ and $B(r)=\left\{s \in \Re^{n}: s>_{L} r\right\}$. It will be shown that $V^{-1}(B(r))$ is open. 
Lemma 1. If $y \succsim x$ for all $y \in V_{1}^{-1}\left(V_{1}(x)\right)$, then $y \succsim x$ for all $y \in X$.

Proof. Suppose $y \succsim x$ for all $y \in V_{1}^{-1}\left(V_{1}(x)\right)$ but there is a $z \in X$ such that $x \succ z$. Since $\succ$ is locally utility representable, there exists $w \in X$ such that $x \succ w$ and $\left.\succ\right|_{\{y \in X: x \succsim y \succ w\}}$ can be represented by a utility function. Therefore there is an $E^{\alpha}$ such that $\left(v_{1}(w), v_{1}(x)\right] \in$ $E^{\alpha}$. By $(1)\left[v_{1}(w), v_{1}(x)\right] \in E^{\alpha}$ which implies $V_{1}(x)=V_{1}(w)$, contradicting the hypothesis that $y \succsim x$ for all $y \in V_{1}^{-1}\left(V_{1}(x)\right)$.

If $V^{-1}(B(r))$ contains no $\succ$-minimal element, then $V^{-1}(B(r))=\bigcup_{x \in V^{-1}(B(r))} B(x)$, which is open.

If $V^{-1}(B(r))$ contains a $\succ$-minimal element which is also a $\succ$-minimal element of $X$, then $V^{-1}(B(r))=X$, which is open.

If $V^{-1}(B(r))$ contains a $\succ$-minimal element $x^{0}$ that is not a $\succ$-minimal element of $X$, then, by Lemma $1, x^{0}$ is not a minimal element of $V_{1}^{-1}\left(V_{1}\left(x^{0}\right)\right)$.

Therefore $V_{1}\left(x^{0}\right)=r_{1}$ and

$V_{1}^{-1}\left(r_{1}\right)-V^{-1}(B(r)) \neq \emptyset$

Since $\left.V_{-1}\right|_{V_{1}^{-1}\left(r_{1}\right)}$ is continuous and $V_{1}^{-1}\left(r_{1}\right) \cap V^{-1}(B(r))=\left(\left.V_{-1}\right|_{V_{1}^{-1}\left(r_{1}\right)}\right)^{-1}(B(r))$,

$V_{1}^{-1}\left(r_{1}\right) \cap V^{-1}(B(r))$ is an open subset of the relative topology on $V_{1}^{-1}\left(r_{1}\right)$

By (5) $V_{1}^{-1}\left(r_{1}\right) \cap V^{-1}(B(r))=O \cap V_{1}^{-1}\left(r_{1}\right)$ where $O$ is open in the $\succ$-order topology on $X$. By (4), there exists $z^{0} \in V_{1}^{-1}\left(r_{1}\right)-V^{-1}(B(r))$. Let $O^{\prime}=O \cap B\left(z^{0}\right)$. Then $O^{\prime}$ is open in the $\succ$-order topology on $X, V_{1}^{-1}\left(r_{1}\right) \cap V^{-1}(B(r))=O^{\prime} \cap V_{1}^{-1}\left(r_{1}\right)$ and $O^{\prime} \cap W\left(x^{0}\right)=\emptyset$. Therefore $O^{\prime} \cup B\left(x^{0}\right)=V^{-1}(B(r))$ so that $V^{-1}(B(r))$ is open.

The following facts establish the continuity of $V: V^{-1}\left(\Re^{n}\right)=X$ is open; $V^{-1}(\emptyset)=\emptyset$ is open; $V^{-1}(B(r))$ has just been shown to be open for all $r \in \Re^{n} ; V^{-1}(W(r))$ is open for all $r \in \Re^{n}$ by a symmetric argument; the sets $B(r)$ and $W(r), r \in \Re^{n}$, form a subbasis for the $>_{L}$-order topology on $\Re^{n}$. 
Proof of Theorem $2(\Rightarrow)$. Suppose $\succ$ has a coordinate-continuous lexicographic representation $v: X \rightarrow \Re^{2}$. Let $Y$ be the set of elements of $X$ at which $\succ$ is exactly one-sided locally utility representable. Let

$Y_{B}=\left\{x \in Y\right.$ : there exists open $O \subseteq X$ such that $x \in O$ and $\left.\succ\right|_{O \cap B(x)}$ can be represented by a utility function\}

Suppose condition (a) does not hold; that is, suppose $Y$ has uncountably many $\sim$-equivalence classes. Without loss of generality, suppose $Y_{B}$ has uncountable many equivalence classes.

If $e$ is a $\sim$-equivalence class of $Y_{B}$ and $e$ is not the set of $\succ$-maximal elements of $X$, choose a representative element $x(e) \in e$ and choose $y(e) \in X-e$ such that $y(e) \succ x(e)$ and $\left.\succ\right|_{B(x(e)) \cap W(y(e))}$ has a utility representation. Let

$$
Z=\left\{x(e) \in Y_{B}: v_{1}(y(e))=v_{1}(x(e))\right\} .
$$

Since $(B(x(e)) \cap W(y(e)) \cap(B(x(f)) \cap W(y(f))=\emptyset$ if $e \neq f$, there can be at most countably many $e$ such that $v_{1}(y(e))>v_{1}(x(e))$ and therefore $Z$ is uncountable.

For positive integer $k$, let

$$
Z^{k}=\left\{x(e) \in Z: v_{2}(y(e)) \geq v_{2}(x(e))+1 / k\right\} .
$$

Since $Z=\bigcup_{k=1}^{+\infty} Z^{k}$ and $Z$ is uncountable, there exists $K$ such that $Z^{K}$ is uncountable. For $e \neq f, v(x(e)) \neq v(x(f))$. Also, for all $y \in X$ and all $x(e) \in Z^{K}$, if $v_{1}(y)=v_{1}(x(e))$, then $v_{2}(y) \geq v_{2}(x(e))$, since $x(e) \in Y_{B}$. These facts imply $v_{1}(x(e)) \neq v_{1}(x(f))$ for $x(e), x(f) \in$ $Z^{K}$ and $e \neq f$. Therefore $v_{1}\left(Z^{K}\right)$ is uncountable.

Since $v_{1}\left(Z^{K}\right)$ is uncountable, there exits $r^{*} \in v_{1}\left(Z^{K}\right)$ and a sequence $<r^{i}>$ in $v_{1}\left(Z^{K}\right)$ such that $r^{i}<r^{*}$ for all $i$ and $r^{i} \rightarrow r^{*}$. If not, for each $r \in v_{1}\left(Z^{K}\right)$ there would exist a nonempty open interval $(s, r) \subseteq \Re$ such that $(s, r) \cap v_{1}\left(Z^{k}\right)=\emptyset$. Then $\left\{(s, r): r \in v_{1}\left(Z^{K}\right)\right\}$ would comprise an uncountable collection of mutually disjoint nonempty open intervals in $\Re$.

There is a unique $x(e) \in Z^{K}$ such that $v_{1}(x(e))=r^{*}$. Choose $<x^{i}>$ and $<y^{i}>$ in $X$ such that $v_{1}\left(x^{i}\right)=v_{1}\left(y^{i}\right)=r^{i}$ for all $i$ and $v_{2}\left(y^{i}\right) \geq v_{2}\left(x^{i}\right)+1 / k$ for all $i$. Since $x(e) \in Y_{B}$, 
$v_{1}\left(x^{i}\right)<v_{1}(x)$ for all $i$ and $v_{1}\left(x^{i}\right) \rightarrow v_{1}(x(e)), x^{i} \rightarrow x(e)$. Similarly, $y^{i} \rightarrow x(e)$. But it is not the case that both $v_{2}\left(x^{i}\right) \rightarrow v_{2}(x(e))$ and $v_{2}\left(y^{i}\right) \rightarrow v_{2}(x(e))$, since $v_{2}\left(y^{i}\right) \geq v_{2}\left(x^{i}\right)+1 / K$ for all $i$.

The assumption that $Y_{B}$ has uncountably many -equivalence classes has contradicted the coordinate continuity of $v$.

It remains to show that condition (b) holds. Let

$$
C=\{x \in X: \succ \text { is locally utility representable at } x\}
$$

A $\succ$-interval of $X$ is a set $S \subseteq X$ such that $x, y \in S$ and $x \succsim y$ together imply $\{z \in$ $X: x \succsim z \succsim y\} \subseteq S$.

Suppose $I$ is a $\succ$-interval of $X,\left.\succ\right|_{I}$ can be represented by a utility function, $I$ is maximal with respect to these two properties and $I \subseteq C$. Let $\left\{I^{\alpha}\right\}$ be the set of all such $I$.

Lemma 2. Each $I^{\alpha}$ contains at least two -equivalence classes-except in the trivial case in which $X$ contains only one $\sim$-equivalence class.

Proof. Suppose $x \in I^{\alpha}, y \in X$ and $\operatorname{not}(x \sim y)$. Without loss of generality suppose $y \succ x$. Since $x \in C$, there exists $z \succ x$ such that $\left.\succ\right|_{B(x) \cap W(z)}$ can be represented by a utility function. Then for the $\succ$-interval $[x, z],\left.\succ\right|_{[x, z]}$ can be represented by a utility function. By the definition of $I^{\alpha}, z \in I^{\alpha}$.

For each $S \subseteq X$, let

$$
l(S)=\sup \left\{\sqrt{\left(v_{1}(a)-v_{1}(b)\right)^{2}+\left(v_{2}(a)-v_{2}(b)\right)^{2}}: a, b \in S\right\} .
$$

By Lemma $2, l\left(I^{\alpha}\right)>0$ for all $I^{\alpha}$ except in the trivial case in which $X$ contains only one $\sim$-equivalence class, which will be ignored from here on.

Suppose $J^{\beta}$ is a $\succ$-interval, $\left.\succ\right|_{J^{\beta}}$ can be represented by a utility function, $J^{\beta}$ is maximal with respect to two properties, and $J^{\beta} \cap Y \neq \emptyset$ (recall that $Y$ is the set of elements of $X$ at which $\succ$ is exactly one-sided locally utility representable). Then $J^{\beta}$ contains one 
or two $\sim$-equivalence classes of $Y$. For each $J^{\beta}$, let $\left\langle J^{\beta}(k)\right\rangle$ be a sequence of closed $\succ$-intervals such that $J^{\beta}(k) \subseteq J^{\beta}(k+1) \subseteq J^{\beta}$ for each $k$ and $J^{\beta}-\bigcup_{k=1}^{+\infty} J^{\beta}(k)=J^{\beta} \cap Y$.

Finally, for $k$ a positive integer, let

$$
A^{k}=(X-C) \cup\left(\cup_{l\left(I^{\alpha}\right)<1 / k} I^{\alpha}\right) \cup\left(\cup_{l\left(J^{\beta}\right)<1 / k} J^{\beta}\right) \cup\left(\cup_{l\left(J^{\beta}\right) \geq 1 / k}\left(J^{\beta}-J^{\beta}(k)\right)\right)
$$

Clearly $<A^{k}>$ is nested; that is, $A^{k+1} \subseteq A^{k}$.

Since $(X-C) \subseteq A^{k}$ for all $k, X-C \subseteq \bigcap_{k=1}^{+\infty} A^{k}$

Suppose $x \in C$. Then $x \in I^{\alpha}$ for some $\alpha$ or $x \in J^{\beta}$ for some $\beta$. If $x \in I^{\alpha}$ choose $K$ such that $1 / K<l\left(I^{\alpha}\right)$. Then $x \notin A^{k}$. If $x \in J^{\beta}, l\left(J^{\beta}\right)>0$ since $J^{\beta}$ also contains an element $y \in Y$. Choose $K$ such that $1 / K<l\left(J^{\beta}\right)$ and $x \in J^{\beta}(K)$. Again $x \notin A^{K}$. Therefore $\bigcap_{k=1}^{+\infty} A^{k} \subseteq X-C$. Since $X-C \subseteq \bigcap_{k=1}^{+\infty} A^{k} \subseteq X-C, X-C=\bigcap_{k=1}^{+\infty} A^{k}$.

It remains to show that each $A^{k}$ is open.

Towards showing that $A^{k}$ is open, suppose $x \in A^{k}$. It will be shown that $x \in O \subseteq A^{k}$ for some open $O$.

Case 1. $x \in I^{\alpha} \subseteq A^{k}$. Since $x \in I^{\alpha}$, there exists open $O \subseteq X$ such that $x \in O$ and $\left.\succ\right|_{O}$ can be represented by a utility function. Since $O$ is open and $\succ$ has a lexicographic representation, there exists $a, b \in X$ such that $x \in B(a) \cap W(b) \subseteq O$ or $x \in B(a) \subseteq O$ or $x \in W(b) \subseteq O$ or the $\sim$-equivalence class of $x$ is $X$. Then $x \in B(a) \cap W(b) \subseteq I^{\alpha}$ or $x \in B(a) \subseteq I^{\alpha}$ or $x \in W(b) \subseteq I^{\alpha}$ or $x \in X=I^{\alpha}$.

Case 2. $x \in J^{\beta} \cap C \subseteq A^{k}$. The argument that $J^{\beta} \cap C$ is open is very similar to the argument in Case 1 that $I^{\alpha}$ is open.

Case 3. $x \in\left(J^{\beta}-J^{\beta}(k)\right) \cap C \subseteq A^{k}$. Since $J^{\beta} \cap C$ is open, $J^{\beta} \cap C-J^{\beta}(k)$ is open.

Case 4. $x \in Y$. Then for some $\beta, x \in J^{\beta}$. Since $x \in Y, y \succsim x$ for all $y \in J^{\beta}$ or $x \succsim y$ for all $y \in J^{\beta}$. Without loss of generality, assume $y \succsim x$ for all $y \in J^{\beta}$. There exists $b \in J^{\beta}$ such that $b \succ x$ and $\{z: b \succ z \succsim x\} \subseteq J^{\beta}-J^{\beta}(k)$, unless $x \succsim y$ for all $y \in X$.

Let

$$
O=\left\{r \in \Re^{2}: \sqrt{\left(v_{1}(x)-r_{1}\right)^{2}+\left(v_{2}(x)-r_{2}\right)^{2}}<1 / 2 k\right\} .
$$

Then by Proposition 1 and the coordinate continuity of $v, v^{-1}(O)$ is open. Since $\left.\succ\right|_{J^{\beta}}$ has a utility function, $y \succsim x$ for all $y \in J^{\beta}$, and $x \in Y,\left.\succ\right|_{v^{-1}(O) \cap W(x)}$ cannot be represented by 
a utility function. Therefore $v^{-1}(O) \cap W(x)$ is not empty. There exists $x^{0} \in v^{-1}(O) \cap W(x)$, such that $B\left(x^{0}\right) \cap W(x) \subseteq v^{-1}(O)$. Then $x^{0} \in X-C-Y$ or $x^{0} \in I^{\alpha^{0}}$ for some $\alpha^{0}$ or $x^{0} \in J^{\beta^{0}}$ for some $\beta^{0}$. Set $S^{0}=\left\{x^{0}\right\}$ or $I^{\alpha^{0}}$ or $J^{\beta^{0}}$ respectively. Again using $x \in Y$, there exists $x^{1} \in v^{-1}(O) \cap W(x)$ such that $x^{1} \succ y$ for all $y \in S^{0}$. If $z \in B\left(x^{1}\right) \cap W(x)$, then $z \in X-C \subseteq A^{k}$; or $z \in I^{\alpha} \subseteq B\left(x^{0}\right) \cap W(x) \subseteq v^{-1}(O) \cap W(x)$ so that $l\left(I^{\alpha}\right)<1 / k$ which implies $I^{\alpha} \subseteq A^{k}$; or $z \in J^{\beta} \subseteq B\left(x^{0}\right) \cap W(x) \subseteq v^{-1}(O) \cap W(x)$ so that $l\left(J^{\beta}\right)<1 / k$ which implies $J^{\beta} \subseteq A^{k}$.

Summarizing the conclusions reached in case 4 , there exist $x^{1}, b \in X$ such that $x \in$ $B\left(x^{1}\right) \cap W(b) \subseteq A^{k}$ or there exists $x^{1} \in X$ such that $x \in B\left(x^{1}\right) \subseteq A^{k}$.

Case 5. $x \in X-C-Y$. Apply the $v^{-1}(O)$ argument in case 4 to both $B(x)$ and $W(x)$.

Proof of Theorem $2(\Leftarrow)$. Suppose $v: X \rightarrow \Re^{2}$ is a lexicographic representation for $\succ$ on $X, Y$ has only countably many $\sim$-equivalence classes and $\left.<A^{k}\right\rangle$ is a nested sequence of open subsets of $X$ such that $\bigcap_{i=1}^{+\infty} A^{K}=X-C$. (Recall that $C$ and $Y$ are the sets of elements of $X$ at which $\succ$ is locally utility representable, and exactly one-sided locally utility representable, respectively).

Construct $V: X \rightarrow \Re^{2}$ as in the proof of Theorem $1(\Leftarrow)$. Recall that

$V$ is a lexicographic representation for $\succ$

for each $x \in X,\left.V_{2}\right|_{V_{1}^{-1}\left(V_{1}(x)\right)}: V_{1}^{-1}\left(V_{1}(x)\right) \rightarrow \Re$ is continuous as a function from

$$
V_{1}^{-1}\left(V_{1}(x)\right) \text { with the } \succ \text {-order topology to } \Re
$$

for $x, y \in X, V_{1}(x)>V_{1}(y)$ implies $\left.\succ\right|_{B(y) \cap W(x)}$ cannot be represented by a utility function

In addition, by Debreu (1964) $V_{1}$ can be taken to be continuous as a function from $X$ with the $\succ_{1}$-order topology to $\Re$, where $\succ_{1}$ is defined by $x \succ_{1} y$ if $V_{1}(x)>V_{1}(y)$. 
Lemma 3. $V_{1}$ is continuous as a function from $X$ with the $\succ$-order topology to $\Re$.

Proof. Suppose $x \in X$. Let $B_{1}(x)=\left\{y \in X: y \succ_{1} X\right\}$. If $y \in B_{1}(x)$, then there exists $z \in X$ such that $y \succ_{1} z \succ_{1} x$, since otherwise $\left.\succ\right|_{B(x) \cap W(y)}$, could be represented by a utility function, contradicting (8). Therefore $y \in B(z) \subseteq B_{1}(x)$. Since $y \in B_{1}(x)$ implies $y \in O \subseteq B_{1}(x)$ for some $O$ open in the order topology, $B_{1}(x)$ is open in the $\succ$-order topology. Similarly $W_{1}(x)$ is open in the $\succ$-order topology. Therefore any subset of $X$ that is open in the $\succ_{1}$-order topology is open in the $\succ$-order topology.

Now suppose $O \subseteq \Re$ is open. Then $V_{1}^{-1}(O)$ is open in the $\succ_{1}$-order topology and therefore open in the $\succ$-order topology.

Unfortunately, despite (7) $V_{2}$ is not necessarily continuous as a function from $X$ with the $\succ$-order topology to $\Re$. A coordinate continuous $w$ will now be constructed. First suppose $J$ is a $\succ$-interval, $\left.\succ\right|_{J}$ can be represented by a utility function, $J$ is maximal with respect to these two properties, and $J \cap Y \neq \emptyset$. Then there exists $x \in Y$ such that $J \cap Y=e(x)$, the $\sim$-equivalence class of $x$, and $y \succsim x$ for all $y \in J$; or there exists $z \in Y$ such that $J \cap Y=e(z)$ and $z \succsim y$ for all $y \in J$; or $J \cap Y=e(x) \cup e(z), z \succ x$ and $z \succsim y \succsim x$ for all $y \in J$.

Since by hypothesis $Y$ has only countable many $\sim$-equivalence classes, there is a countable collection $\left\{J^{i}\right\}$ of all $J$ 's described above.

Construction of $w: X \rightarrow \Re^{2}$.

Step 1. Construct a countable collection $\left\{\left[a^{i}, b^{i}\right]\right\}$ of mutually disjoint closed intervals in $\Re$ and a function $f: \Re-\left(\cup_{i} V_{1}\left(J^{i}\right)\right) \rightarrow \Re-\left(\cup_{i}\left[a^{i}, b^{i}\right]\right)$ such that $f$ is continuous, $1-1$, onto and order preserving.

Step 2. For each $J^{i}$, let $g^{i}:\left[\inf V_{2}\left(J^{i}\right), \sup V_{2}\left(J^{i}\right)\right] \rightarrow\left[a^{i}, b^{i}\right]$ be a continuous, 1-1, onto, order preserving function. 
Step 3. Define $w_{1}: X \rightarrow \Re$ by

$$
w_{1}(x)= \begin{cases}g^{i}\left(V_{2}(x)\right) & \text { if } x \in J^{i} \\ f\left(V_{1}(x)\right) & \text { if } \left.x \in X-\left(\cup_{i} J^{i}\right)\right) .\end{cases}
$$

Step 4. Let $\left\{I^{\alpha}\right\}$ be the collection of all $I$ such that $I$ is a $\succ$-interval, $\left.\succ\right|_{I}$ can be represented by a utility function, $I$ is maximal with respect to these two properties, and $I \subseteq C$.

For each $I^{\alpha}$, define a continuous, onto, order preserving function

$$
h^{\alpha}:\left[\inf V_{2}\left(I^{\alpha}\right), \sup V_{2}\left(I^{\alpha}\right)\right] \rightarrow\left[0, \max \left\{1 / k: \operatorname{not}\left(I^{\alpha} \subseteq A^{k}\right)\right\}\right]
$$

Step 5. Finally, define $w_{2}: X \rightarrow \Re$ by

$$
w_{2}(x)= \begin{cases}0 & \text { if } x \in X-\left(\cup_{\alpha} I^{\alpha}\right) ; \\ h^{\alpha}\left(V_{2}(x)\right) & \text { if } x \in I^{\alpha} .\end{cases}
$$

It remains to show that $w=\left(w_{1}, w_{2}\right)$ is a coordinate continuous lexicographic representation for $\succ$.

That $w$ is a lexicographic representation is clear from (6) and the construction of $w$ from $V$ in such a way that $w(x)>_{L} w(y)$ if and only if $V(x)>_{L} V(y)$.

That $w_{1}$ is continuous follows from Lemma 3 , and the construction of $w_{1}$.

Next suppose $O \subseteq \Re$ is open and $x \in w_{2}^{-1}(O)$. Now $w_{2}$ will be proven continuous by producing $U \subseteq X$ such that $x \in U \subseteq w_{2}^{-1}(O)$ and $U$ is open in the $\succ$-order topology on $X$.

Case 1. $x \in I^{\alpha}$ for some $\alpha$. By (7) and the continuity of $h^{\alpha}, w_{2}^{-1}(O) \cap I^{\alpha}$ is open in the $\succ$-order topology on $I^{\alpha}$. Therefore $w_{2}^{-1}(O) \cap I^{\alpha}=A \cap I^{\alpha}$ where $A \subseteq X$ is open in the $\succ$-order topology on $X$. Since $I^{\alpha}$ is also open in the $\succ$-order topology on $X, A \cap I^{\alpha}$ is open in the $\succ$-order topology on $X$. Let $U=A \cap I^{\alpha}$. Then $x \in w_{2}^{-1}(O) \cap I^{\alpha}=U \subseteq w_{2}^{-1}(O)$.

Case 2. $x \in J^{\beta}-Y$ for some $\beta$. Since $w_{2}\left(J^{\beta}-Y\right)=\{0\}, w_{2}(x)=0$ and $x \in w_{2}^{-1}(O)$, $J^{\beta}-Y \subseteq w_{2}^{-1}(O)$. Also $J^{\beta}-Y$ is open. Let $U=J^{\beta}-Y$.

Case 3. $x \in J^{\beta} \cap Y$ for some $\beta$. Without loss of generality, assume $x \in Y^{\beta}$. Recall that $x \in Y^{\beta}$ implies $y \succsim x$ for all $y \in J^{\beta}$. Choose $k$ such that $[0,1 / k] \subseteq O$. Since 
$x \in Y^{\beta}$ and $A^{k}$ is open, there exists $x^{0}, x^{1} \in X$ such that $w_{1}(x)>w_{1}\left(x^{1}\right)>w_{1}\left(x^{0}\right)$ and $B\left(x^{0}\right) \cap W(x) \subseteq A^{k}$. For $y \in X$ such that $x \succ y \succ x^{1}$ either $y \in X-\cup I^{\alpha}$ so that $w_{2}(y)=0$ or $y \in I^{\alpha}$. If $y \in I^{\alpha}$, then since $B\left(x^{0}\right) \cap W(x) \subseteq A^{k}$, it must be that $I^{\alpha} \subseteq A^{k}$. Since $I^{\alpha} \subseteq A^{k}, w_{2}(y) \in[0,1 / k] \subseteq O$. Let

$$
U=\left(B\left(x^{1}\right) \cap W(x)\right) \cup w_{1}^{-1}\left(w_{1}(x)\right) \cup\left(J^{\beta}-Y\right)
$$

Then $x \in U \subseteq w_{2}^{-1}(O)$ and $U$ is open.

Case 4. $x \in X-C-Y$. Construct $x^{1}$ as in case 3 and construct $x^{2}$ symmetrically, so that $w_{1}\left(x^{2}\right)>w_{1}(x)$ and if $y \in W\left(x^{2}\right) \cap B(x)$, then $w_{2}(y) \in O$. Let $U=B\left(x^{1}\right) \cap W\left(x^{2}\right)$. 


\section{References}

[1] Binmore, K. and L. Samuelson, (1992) "Evolutionary Stability in Repeated Games Played by Finite Automata," Journal of Economic Theory 57 (2), 278-305.

[2] Debreu, G., (1964) "Continuity Properties of Paretian Utility," International Economic Review 5, 285-293.

[3] Dugundji, J. (1966) Topology, Allyn and Bacon Inc., Boston.

[4] Fishburn, P., (1975) "Axioms for Lexicographic Preferences" Review of Economic Studies 42 (3), 415-419.

[5] Hougaard, J.L, and M. Tvede (2000) "The Existence of Maximal Elements: Generalized Lexicographic Relations," Journal of Mathematical Economics 36, 111-115.

[6] Jensen,E., (1990) "An Econometric Analysis of the Old-Age Security Motive for Childbearing," International Economic Review 31 (4), 953-968.

[7] Knoblauch, V., (2000) "Lexicographic Orders and Preference Representation," Journal of Mathematical Economics 34, 255-267. 